Check for updates

Cite this: RSC Adv., 2019, 9, 25488

Received 22nd June 2019

Accepted 8th August 2019

DOI: $10.1039 / c 9 r a 04676 c$

rsc.li/rsc-advances

\section{Bismuth trioxide-tailored sintering temperature, microstructure and NTCR characteristics of $\mathrm{Mn}_{1.1} \mathrm{Co}_{1.5} \mathrm{Fe}_{0.4} \mathrm{O}_{4}$ ceramics}

\begin{abstract}
Bing Wang, (D) ${ }^{\text {ab Junhua Wang, }}$ ab Aimin Chang ${ }^{\text {ab }}$ and Jincheng Yao (D) *ab
$\mathrm{Mn}_{1.1} \mathrm{CO}_{1.5} \mathrm{Fe}_{0.4} \mathrm{O}_{4}$ ceramics with tailored sintering temperature, microstructure, and NTCR characteristics were prepared using $\mathrm{Bi}_{2} \mathrm{O}_{3}$ sintering additive by a solid-state reaction route. Densification and morphological characterization indicate that bismuth trioxide can play a critical role in the sintering process. The results reveal that the sintering temperature can be decreased significantly from $1200{ }^{\circ} \mathrm{C}$ to $1050{ }^{\circ} \mathrm{C}$ by using the appropriate content of $\mathrm{Bi}_{2} \mathrm{O}_{3}$ additive. The resistivity decreases first and then increases with increasing $\mathrm{Bi}_{2} \mathrm{O}_{3}$ content. The obtained $B_{25 / 50}$ value and $\rho_{25}$ ranges were $3647-3697 \mathrm{~K}$, and 800-1075 $\Omega \mathrm{cm}$, respectively. Oxygen sorption theory can be used to illustrate the optimal thermal stability $\left(\Delta R / R_{0}=0.10 \%\right)$. Complex impedance analysis further elucidates that grain boundaries make a dominant contribution to the total resistance. The mechanisms of grain boundary conduction and relaxation behavior are systematically analyzed. These findings open up a window for the further advancement of NTC ceramics at lower sintering temperature.
\end{abstract}

\section{Introduction}

With the accelerated development of electronic components for miniaturization and integration, multilayer chip thermistors have been widely used in electronic circuits as they facilitate the integration of different electronic devices. Consequently, the assembly of low temperature co-fired ceramics (LTCC) with discrete sensors, resistors, and inductors into a single monolithic chip component can achieve multifunctional performance and promote miniaturization in the printed circuit board (PCB) during assembly. ${ }^{1-4}$ For example, Wang et al. ${ }^{5}$ reported a novel V-modified $\mathrm{Li}_{3} \mathrm{Mg}_{2} \mathrm{NbO}_{6}$ ceramics with outstanding microwave dielectric properties and can reach densification at $900{ }^{\circ} \mathrm{C}$. Li et al. ${ }^{6}$ synthesized the In-doped $\mathrm{Mg}^{-}$ Cd ferrite at low sintering temperature using $\mathrm{Bi}_{2} \mathrm{O}_{3}$ additive. These efforts make these ceramics promising candidates for LTCC applications. Negative temperature coefficient resistors (NTCR) are ceramic semiconductor components whose resistance decreases with increasing temperature. Many material systems have used for NTC ceramics, including stable Mn-based ternary systems, e.g., $\mathrm{Mn}-\mathrm{Co}-\mathrm{Fe}-\mathrm{O}, \mathrm{Mn}-\mathrm{Co}-\mathrm{Ni}-\mathrm{O}, \mathrm{Mn}-\mathrm{Cu}-\mathrm{Ni}-$ $\mathrm{O}$, and others. Due to the characteristics of fast response, high sensitivity, and low cost, $\mathrm{Mn}-\mathrm{Co}-\mathrm{Fe}-\mathrm{O}$ NTC ceramics are promising materials for chip and LTCC applications. ${ }^{7-10}$

${ }^{a}$ Key Laboratory of Functional Materials and Devices for Special Environments of CAS, Xinjiang Key Laboratory of Electronic Information Materials and Devices, Xinjiang Technical Institute of Physics \& Chemistry of CAS, 40-1 South Beijing Road, Urumqi, China.E-mail:yaojc@ms.xjb.ac.cn

${ }^{b}$ University of Chinese Academy of Sciences, Beijing 100049, China
However, the high sintering temperature has been one of the main problems. In previous studies, the sintering temperature of Mn-Co-Fe-O NTC ceramics is typically at and above $1200{ }^{\circ} \mathrm{C}$, which will cause the high production cost and difficult quality control. ${ }^{10-12}$

To date, numerous successful methods for decreasing the sintering temperature have demonstrated with $\mathrm{Ni}-\mathrm{Zn}$ ferrites and $\mathrm{BaTiO}_{3}$ systems, ${ }^{13-16}$ but the development of NTC ceramics is in its infancy. ${ }^{17}$ Therefore, studying the decreasing of the sintering temperature of $\mathrm{Mn}-\mathrm{Co}-\mathrm{Fe}-\mathrm{O}$ NTC ceramics is of great importance. Three main methods have been proposed to decrease the sintering temperature: using materials with fine powders, doping with sintering aids, and chemical processing. ${ }^{18-20}$ Sintering aid by liquid mass transfer mechanism or solution is valuable for decreasing the sintering temperature. For instance, Ye et al. ${ }^{21}$ using $\mathrm{Si}$ as a sintering aid in $\mathrm{B}_{4} \mathrm{C}$ ceramics and found that the relative density can reach $99 \%$ for the formation of liquid Si during sintering.

In previous studies, $\mathrm{Bi}_{2} \mathrm{O}_{3}$ has been demonstrated to be a useful sintering aid in some materials. For example, He et al. ${ }^{22}$ fabricated compact $\mathrm{SrBi}_{2} \mathrm{Nb}_{2} \mathrm{O}_{9-x} \mathrm{Bi}_{2} \mathrm{O}_{3}$ ceramics and obtained excellent electrical properties, e.g., $\rho_{\mathrm{rd}}=96.4 \%, d_{33}=18 \mathrm{pC}$ $\mathrm{N}^{-1}, 2 P_{\mathrm{r}}=17.8 \mathrm{mC} \mathrm{cm}^{-2}$ and $T_{\mathrm{c}}=420^{\circ} \mathrm{C}$. Xu et al. ${ }^{23}$ synthesized dense NiCuZn ferrite ceramics with small grains and sintered at $875{ }^{\circ} \mathrm{C}$ via changing the amount of $\mathrm{Bi}_{2} \mathrm{O}_{3}$ and $\mathrm{Nb}_{2} \mathrm{O}_{5}$ additives. However, the effects of $\mathrm{Bi}_{2} \mathrm{O}_{3}$ addition on the sinterability of NTC ceramics have not studied. In this study, liquid- $\mathrm{Bi}_{2} \mathrm{O}_{3^{-}}$ tailored sintering temperature, microstructure and NTCR Characteristics of $\mathrm{Mn}_{1.1} \mathrm{Co}_{1.5} \mathrm{Fe}_{0.4} \mathrm{O}_{4}$ ceramics have investigated. 


\section{Experimental}

\subsection{Experimental procedures}

Analytical grade $\mathrm{Co}_{2} \mathrm{O}_{3}, \mathrm{Mn}_{3} \mathrm{O}_{4}$, and $\mathrm{Fe}_{2} \mathrm{O}_{3}$ powders were weighed in their respective stoichiometric ratios to synthesize $\mathrm{Mn}_{1.1} \mathrm{Co}_{1.5} \mathrm{Fe}_{0.4} \mathrm{O}_{4}$ (MCF) ceramics. The mixture of weighed powders, deionized water, and alcohol was ball-milled for $10 \mathrm{~h}$ in the Teflon jar using $\mathrm{ZrO}_{2}$ as the grinding medium. The obtained slurry was dried at $85{ }^{\circ} \mathrm{C}$ in an oven for $20 \mathrm{~h}$. Then the dried powders were ground in an agate mortar and calcinated at $950{ }^{\circ} \mathrm{C}$ for $3 \mathrm{~h}$. Then, $x$ wt $\% \mathrm{Bi}_{2} \mathrm{O}_{3}$ (where $x=0,0.05,0.1,0.15$ ) additives were added into the calcined powders and ball-milled for $12 \mathrm{~h}$ using alcohol as the medium. The as-obtained slurry was dried at $85{ }^{\circ} \mathrm{C}$ for $20 \mathrm{~h}$ and then ground. Hereafter, the asobtained powders pressed into the pellet with $10 \mathrm{~mm}$ diameter and the thickness about $2 \mathrm{~mm}$ at $1.2 \mathrm{MPa}$. Then the pellet was pressed using cold isostatic pressing (CIP) at $300 \mathrm{MPa}$ for $120 \mathrm{~s}$ dwell time. The green bodies were sintered at different temperatures $\left(T=1200{ }^{\circ} \mathrm{C}, 1150{ }^{\circ} \mathrm{C}, 1100{ }^{\circ} \mathrm{C}, 1050{ }^{\circ} \mathrm{C}, 1000{ }^{\circ} \mathrm{C}\right)$ for a holding time of $4 \mathrm{~h}$. The silver paste coated on the two opposite sides as the electrode of the sintered discs to investigate the electrical properties. Then the discs were heated to $835{ }^{\circ} \mathrm{C}$ for $20 \mathrm{~min}$ and quenched to achieve the metallization. Subsequently, ten samples per set for measuring electrical resistance held with a holder in a bath of silicone oil.

\subsection{Measurements and characterization}

The crystal structure of the samples determined by the X-ray diffraction (XRD) (Bruker D8 Advance, Germany) patterns with $\mathrm{Cu} \mathrm{K} \alpha$ radiation. The Archimedes technique was used to measure the bulk density to characterize the densification of the samples. The microstructures of the as-sintered samples were examined using a ZEISS SUPRA55VP scanning electron microscope (SEM). The distributions of constituent elements were confirmed using energy dispersive spectroscopy (EDS) (Bruker X-flash5010, Germany). For the electrical properties, the fourterminal method was carried out to measure the electrical resistance using the Agilent 34401A digital multimeter in the range of $-75{ }^{\circ} \mathrm{C}$ to $55^{\circ} \mathrm{C}$. The aging test conducted at $125^{\circ} \mathrm{C}$ for $300 \mathrm{~h}$ in the air. The material constant ( $B$ value), and the aging coefficient $\left(\Delta R / R_{0}\right)$ can be calculated using the following equations:

$$
\begin{gathered}
B=\frac{\ln \rho_{1} / \rho_{2}}{1 / T_{1}-1 / T_{2}} \\
\Delta R / R_{0}=\frac{R-R_{0}}{R_{0}} \times 100 \%
\end{gathered}
$$

where $\rho_{1}$ and $\rho_{2}$ are the resistance values at the absolute temperature of $T_{1}$ and $T_{2}$, respectively, $k$ is the Boltzmann constant, and $R$ and $R_{0}$ are the resistance before and after aging.

The as-sintered ceramics were analyzed using an impedance analyzer (HP41494A). The real $\left(Z^{\prime}\right)$ and imaginary parts $\left(Z^{\prime \prime}\right)$ of complex impedance can be calculated as:

$$
\begin{gathered}
Z^{\prime}=R_{\mathrm{b}}+\frac{R_{\mathrm{gb}}}{1+\omega^{2} C_{\mathrm{gb}}{ }^{2} R_{\mathrm{gb}}{ }^{2}} \\
Z^{\prime \prime}=\frac{\omega C_{\mathrm{gb}} R_{\mathrm{gb}}{ }^{2}}{1+\omega^{2} C_{\mathrm{gb}}{ }^{2} R_{\mathrm{gb}}{ }^{2}}
\end{gathered}
$$

where $\omega(\omega=2 \pi f)$ is the angular frequency, $C_{\mathrm{gb}}$ and $R_{\mathrm{gb}}$ are the grain boundaries' capacitance and resistance and $R_{\mathrm{b}}$ to the bulk.

\section{Results and discussions}

\subsection{XRD patterns}

Fig. 1 shows the XRD patterns of the MCF ceramics sintered at $1050{ }^{\circ} \mathrm{C}$ for $4 \mathrm{~h}$. The XRD results show that all samples mainly contained the cubic spinel phase and no other peaks or peak drift were observed, indicating that the $\mathrm{Bi}_{2} \mathrm{O}_{3}$ addition did not interfere with the phase composition of the MCF ceramics. Due to the bismuth ion has a radius of $1.03 \AA$ ( 6 coordination), which is larger than that of manganese, iron and cobalt ions, about 0.6 $\AA$, so it's difficult to enter the lattice of the spinel. However, the amount of $\mathrm{Bi}_{2} \mathrm{O}_{3}$ is too small, which makes XRD hard to detect. As a result, the XRD patterns have not changed.

\subsection{Densification and microstructure analysis}

Fig. 2 shows the variation tendency of bulk density and sintering temperature of MCF ceramics as a function of $\mathrm{Bi}_{2} \mathrm{O}_{3}$ content. As shown in Fig. 2, the bulk density has no apparent changes when the sintering temperatures higher than $1100{ }^{\circ} \mathrm{C}$. Interestingly, the addition of $\mathrm{Bi}_{2} \mathrm{O}_{3}$ at $1000{ }^{\circ} \mathrm{C}$ and $1050{ }^{\circ} \mathrm{C}$ had a rather notable effect on the bulk density of the MCF ceramics, i.e., the bulk density increased with the increased amount of $\mathrm{Bi}_{2} \mathrm{O}_{3}$ additive by a large amount. Unfortunately, the maximum bulk density at $1000{ }^{\circ} \mathrm{C}$ only reached $4.409 \mathrm{~g} \mathrm{~cm}^{-3}$, which failed to reach the densification condition. On the contrary, the bulk density considerably increased from $4.457 \mathrm{~g} \mathrm{~cm}^{-3}$ to $5.074 \mathrm{~g} \mathrm{~cm}^{-3}$ when the samples sintered at $1050{ }^{\circ} \mathrm{C}$, which can

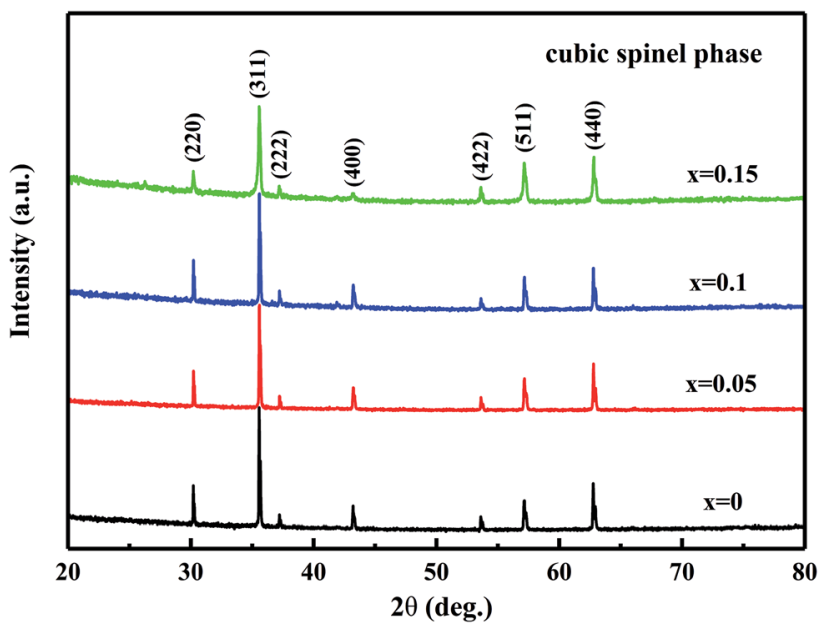

Fig. 1 XRD patterns of MCF samples with $x$ wt\% $\mathrm{Bi}_{2} \mathrm{O}_{3}(0 \leq x \leq 0.15)$ sintered at $1050{ }^{\circ} \mathrm{C}$ for $4 \mathrm{~h}$ 


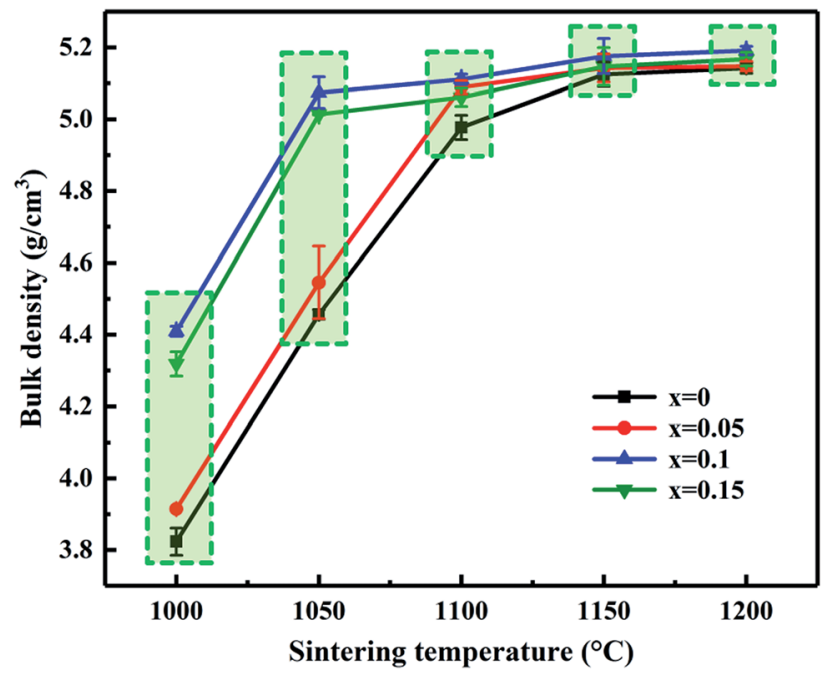

Fig. 2 Bulk density and sintering temperature of MCF ceramics as a function of $\mathrm{Bi}_{2} \mathrm{O}_{3}$ content $(0-0.15 \mathrm{wt} \%)$.

obtain the dense MCF ceramics. Therefore, the $1050{ }^{\circ} \mathrm{C}$ selected as the sintering temperature for further study. Besides, the bulk density increased with the addition of $\mathrm{Bi}_{2} \mathrm{O}_{3}$ compared to that of the initial value. However, as the $\mathrm{Bi}_{2} \mathrm{O}_{3}$ content further increased, the bulk density decreased at a constant temperature. It attributed to the appearance of liquid phase improves the transport rates of grain coarsening, so grain growth mixed with some pores occurred.
Fig. 3 shows the SEM micrographs of MCF ceramics with $x$ wt $\% \mathrm{Bi}_{2} \mathrm{O}_{3}$ contents $(0 \leq x \leq 0.15)$ sintered at $1050^{\circ} \mathrm{C}$. In the sample without $\mathrm{Bi}_{2} \mathrm{O}_{3}$ (Fig. 3a), the sintering temperature of $1050{ }^{\circ} \mathrm{C}$ did not achieve the densification condition. As such, many pores observed along with heterogeneous grains. As the $\mathrm{Bi}_{2} \mathrm{O}_{3}$ content increased from 0 to $0.1 \mathrm{wt} \%$ (Fig. $3 \mathrm{a}$ and c), some pores disappeared, while the densification rate and grain homogeneity increased by a large amount. This transformation can be attributed to the appearance of a trace amount of liquid layer at the grain boundaries, providing a capillary force and driving force to reduce the interfacial free energy of the system that pulls the grains together which promotes grain densification and homogeneity. ${ }^{24,25}$ However, as the $\mathrm{Bi}_{2} \mathrm{O}_{3}$ content further increased (Fig. 3d), the grain size gradually increased and the homogeneity became poor.

A theoretical model of the microstructure evolutions during the sintering process proposed as depicted in Fig. $4 .{ }^{24,26}$ In the initial state, it can observe loose small grains mixed with bismuth trioxide additives. As the increase of temperature, $\mathrm{Bi}_{2} \mathrm{O}_{3}$ additives begin to soften and turn into the liquid phase. Meanwhile, a rearrangement of particles happened. As the temperature rises further, two different mechanisms started to appear, i.e., densification and grain coarsening in the final stage of the sintering. ${ }^{26}$ Capillary forces and the driving force to reduce the interfacial free energy of the system, especially, $\Delta G$ (liquid-vapor) - create shear and rotational actions of particles diminishing the pores by transitioning from loose packing to close packing. Hence, dense microstructures may occur. ${ }^{27}$
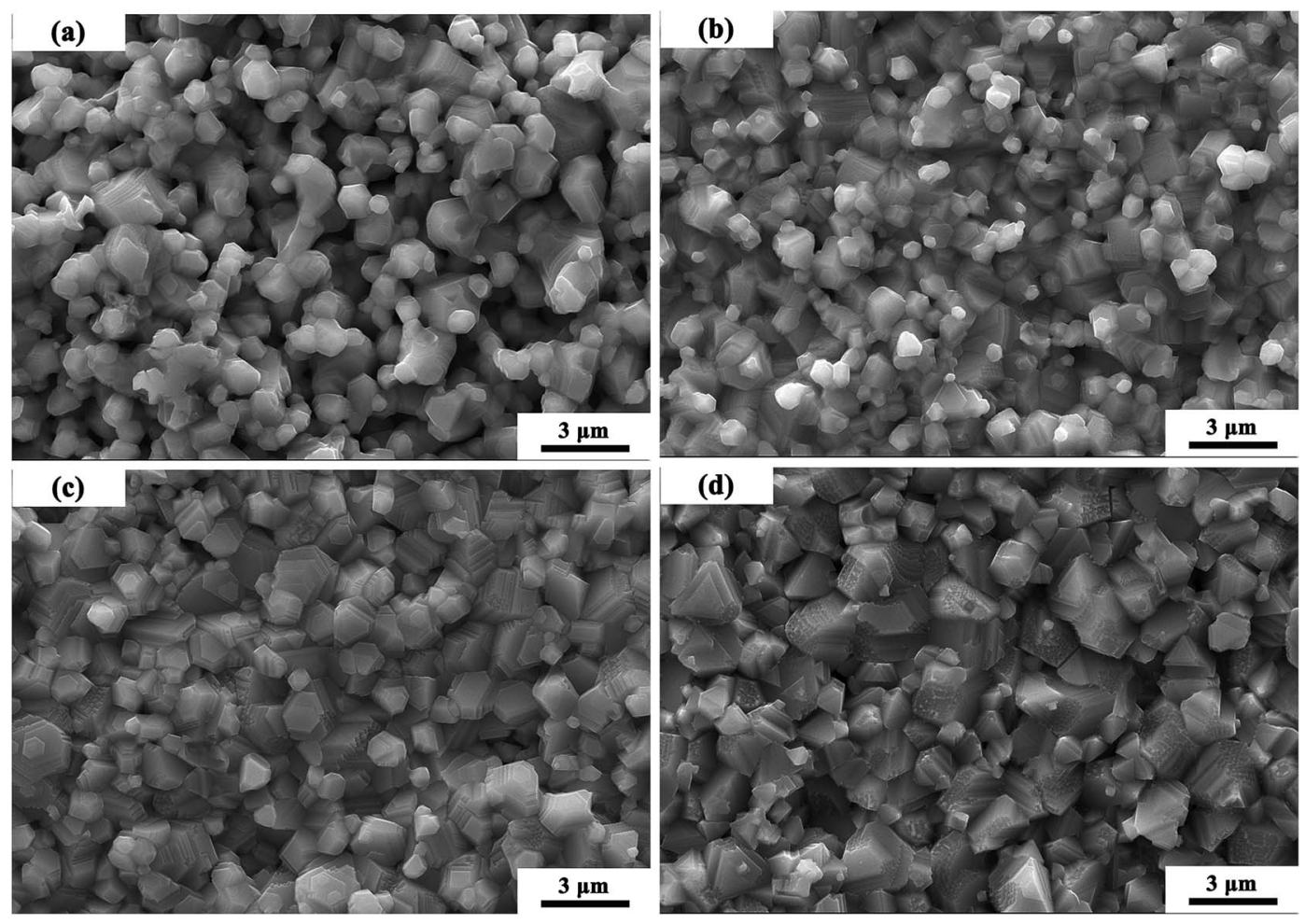

Fig. 3 SEM micrographs of MCF ceramics with $x$ wt $\% \mathrm{Bi}_{2} \mathrm{O}_{3}$ contents $(0 \leq x \leq 0.15)$ sintered at $1050{ }^{\circ} \mathrm{C}:(\mathrm{a}) x=0,(\mathrm{~b}) x=0.05,(\mathrm{c}) x=0.10,(\mathrm{~d}) x=$ 0.15 . 

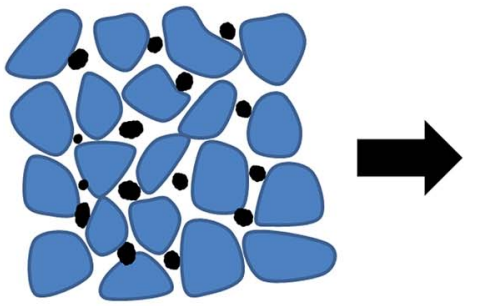

Initial state: mixed powders
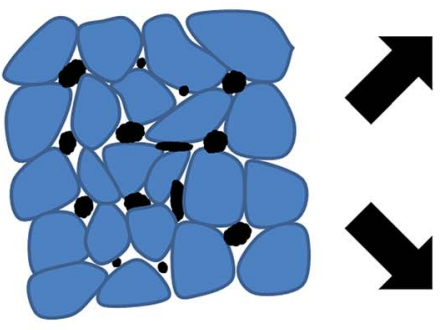

Rearrangement

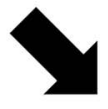

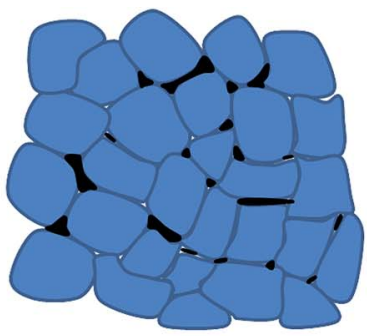

Densification

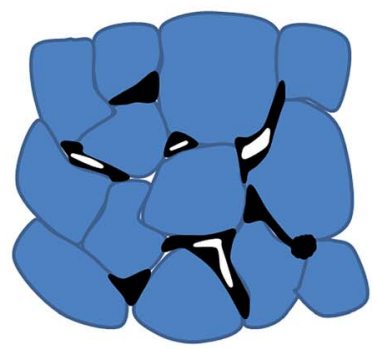

Grain coarsening

Fig. 4 Schematic diagram of the liquid- $\mathrm{Bi}_{2} \mathrm{O}_{3}$-assisted sintering of MCF ceramics.

Besides, the appearance of the liquid phase can improve the transport rates of grain coarsening, so grain growth mixed with some pores may happen. At this stage, the amount of additive and sintering temperature are crucial for densification and grain coarsening. ${ }^{28,29}$

SEI and elemental maps were obtained to determine the distribution of the constituent elements in the samples. In this sample, the $\mathrm{Mn}$, Co, Fe, and Bi distributions were homogeneous, while those of $\mathrm{O}$ were heterogeneous. Some oxygen- deficient regions were observed at the grain boundaries. Representative SEI and elemental maps of the MCF samples with 0.15 wt $\% \mathrm{Bi}_{2} \mathrm{O}_{3}$ sintered at $1050{ }^{\circ} \mathrm{C}$ are shown in Fig. 5 .

\subsection{Electrical properties}

Fig. 6 shows the specific resistance $(\rho)$-temperature $(T)$ dependence relationship from $-75{ }^{\circ} \mathrm{C}$ to $55{ }^{\circ} \mathrm{C}$ of the MCF ceramics sintered at $1050^{\circ} \mathrm{C}$. The specific resistance of each sample each
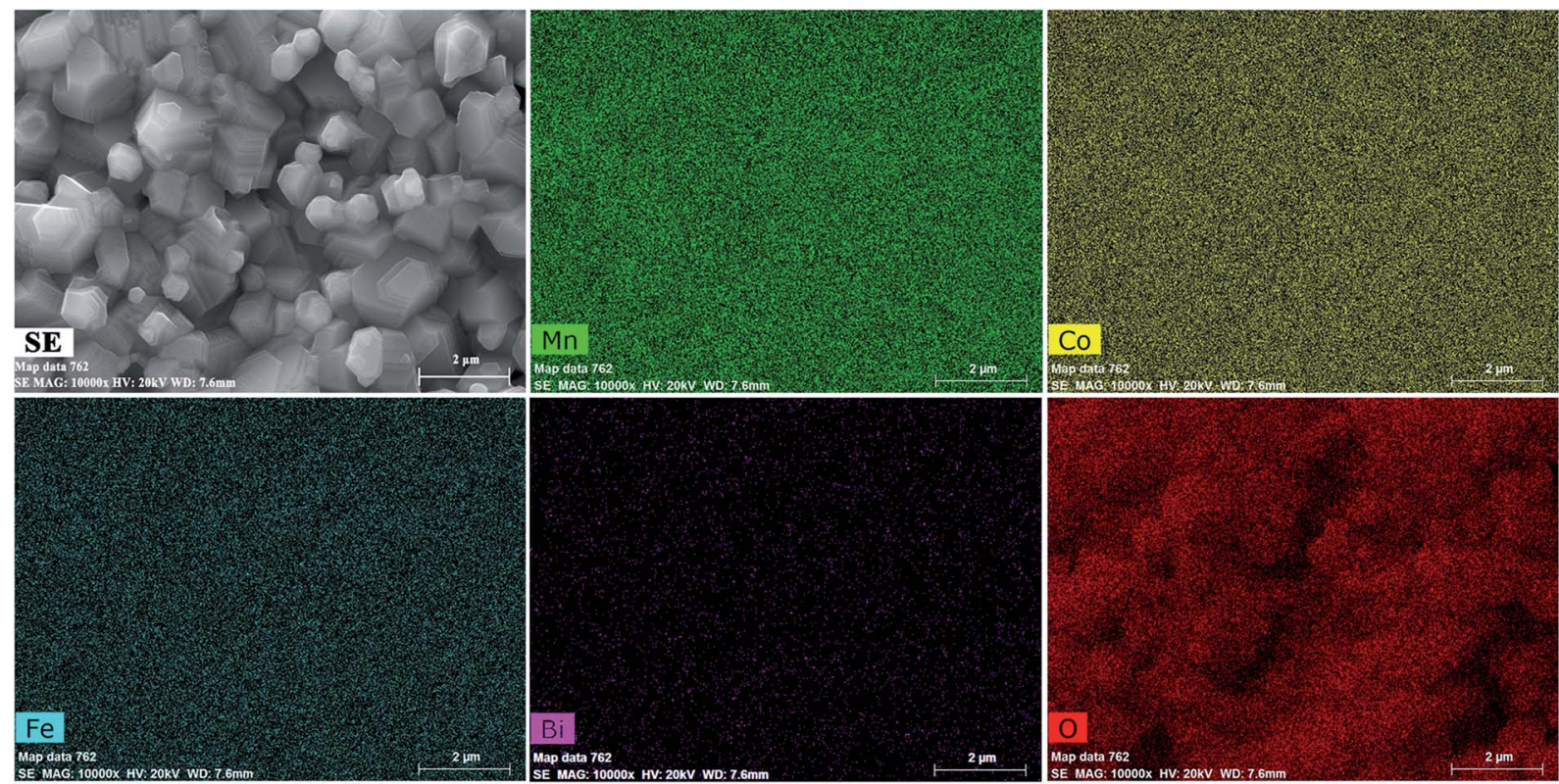

Fig. $5 \mathrm{SEl}$ and maps of elements $\mathrm{Mn}$, Co, $\mathrm{Fe}, \mathrm{Bi}$, and $\mathrm{O}$ for MCF samples with 0.15 wt $\% \mathrm{Bi}_{2} \mathrm{O}_{3}$ sintered at $1050{ }^{\circ} \mathrm{C}$. 


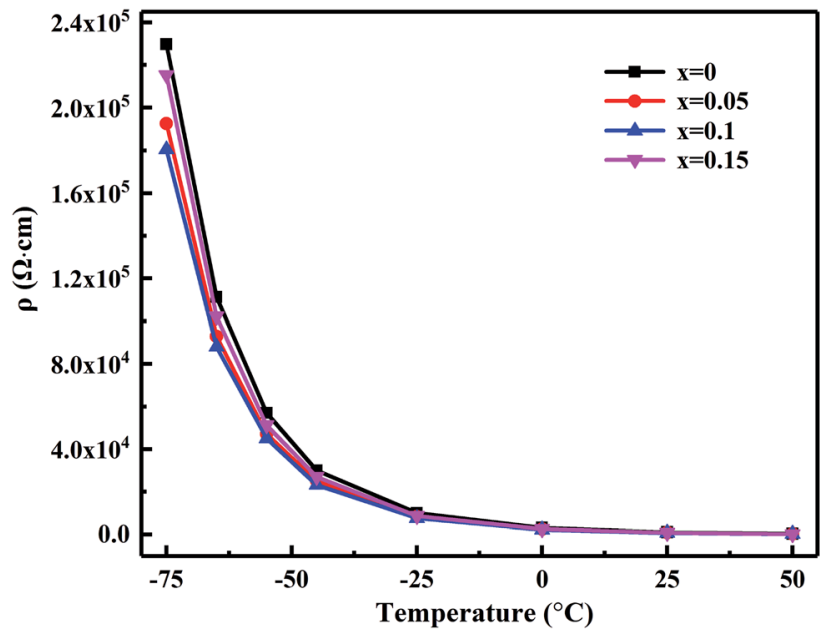

Fig. 6 Plots of the $\rho$ vs. $T$ for the MCF ceramics with $x$ wt $\% \mathrm{Bi}_{2} \mathrm{O}_{3}$ contents $(0 \leq x \leq 0.15)$ sintered at $1050^{\circ} \mathrm{C}$.

sample decreased exponentially with increasing temperature, corresponding to typical NTC characteristics.

Fig. 7 shows the material constant $B_{25 / 50}$ and resistivity $\rho_{25}$ as a function of $\mathrm{Bi}_{2} \mathrm{O}_{3}$ content for MCF ceramics. The obtained $\rho_{25}$ and $B_{25 / 50}$ range from 800 to $1075 \Omega \mathrm{cm}$ and 3647 to $3697 \mathrm{~K}$, respectively. Also, the specific resistance decreased upon addition of $\mathrm{Bi}_{2} \mathrm{O}_{3}$ from 0 to $0.1 \mathrm{wt} \%$. When the $\mathrm{Bi}_{2} \mathrm{O}_{3}$ content increased to $0.15 \mathrm{wt} \%$, however, an increase in specific resistance occurred. Since the resistivity of $\mathrm{Bi}_{2} \mathrm{O}_{3}$ is $10^{6} \Omega \mathrm{cm}$ or more, the electrical resistivity of the sintered body is considered to increase as the $\mathrm{Bi}_{2} \mathrm{O}_{3}$ content increases. However, the electrical resistivity of the sintered body decreased upon addition of $\mathrm{Bi}_{2} \mathrm{O}_{3}$ from 0 to $0.1 \mathrm{wt} \%$. This can be attributed to the following two reasons: (1) it is well known that the electrons move through the shortest contact point between the oxide particles. Therefore, the increased densification reduces the electron transport distance between the $\mathrm{Mn}^{3+}$ and $\mathrm{Mn}^{4+}$. In other words, conduction caused by the hopping electrons between $\mathrm{Mn}^{3+}$ and $\mathrm{Mn}^{4+}$ is easier on octahedral and, thus, a decrease in the resistivity; (2)

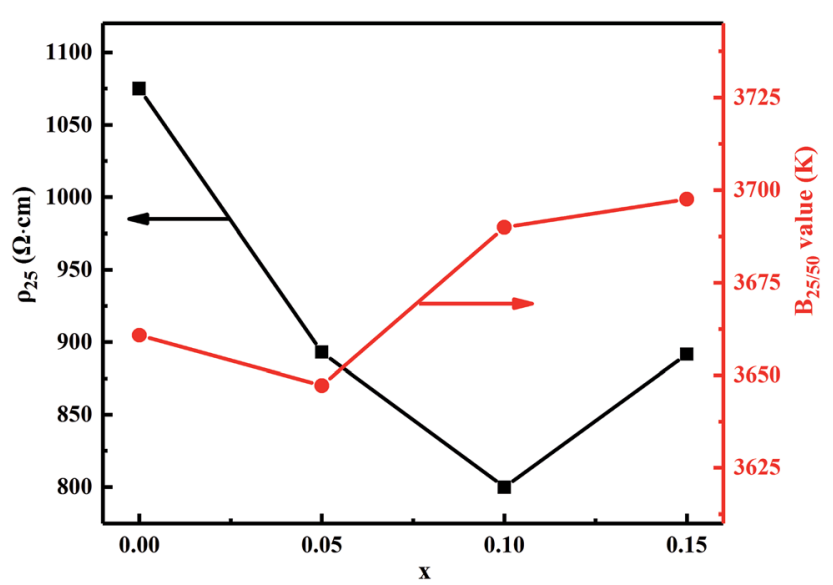

Fig. 7 Material constant $B$ and specific resistivity $\rho_{25}$ of MCF ceramics sintered at $1050{ }^{\circ} \mathrm{C}$. due to bismuth oxide has relatively low ionization energy, upon addition of $\mathrm{Bi}_{2} \mathrm{O}_{3}$ from 0 to $0.1 \mathrm{wt} \%$, the ionization energy decreased, which increased the concentration of carriers capable of accepting or donating electrons during transfer and, thereby, decreasing the resistivity. Besides, it also can notice that $B_{25 / 50}$ constant decreases first and then increases as the $\mathrm{Bi}_{2} \mathrm{O}_{3}$ increases. It mainly results from the variation in activation energy causes the change of jumping resistance between $\mathrm{Mn}^{3+}$ and $\mathrm{Mn}^{4+}$ ions.

Fig. 8 shows the relative resistance drift $\Delta R / R_{0}$ as a function of the $\mathrm{Bi}_{2} \mathrm{O}_{3}$ content for MCF ceramics after the aging test at $125{ }^{\circ} \mathrm{C}$ for $300 \mathrm{~h}$. The as-obtained ceramics exhibited excellent thermal stability. The appropriate content of $\mathrm{Bi}_{2} \mathrm{O}_{3}$ strongly decreased the resistance drift from 2.47 to $0.10 \%$. The minimum resistance drift $\left(\Delta R / R_{0}=0.10 \%\right)$ obtained with the $0.1 \mathrm{wt} \% \mathrm{Bi}_{2} \mathrm{O}_{3}$ addition. In contrast, when the $\mathrm{Bi}_{2} \mathrm{O}_{3}$ content increased to $0.15 \mathrm{wt} \%$, an increase in electrical drift was observed. It illustrated by the following reason.

Based on the densification and SEM analysis above, upon the addition of $\mathrm{Bi}_{2} \mathrm{O}_{3}$ from 0 to $0.1 \mathrm{wt} \%$, the increased densification and optimized microstructure stabilized the configurations of the cation distribution. It inhibits the oxidation and diffusion of the cationic vacancies between the grains and the grain boundaries, leading to a decreasing of resistivity drift. However, $0.15 \mathrm{wt} \% \mathrm{Bi}_{2} \mathrm{O}_{3}$ content resulted in grain coarsening and decreased densification, more atmospheric oxygen sorption has appeared following the defect reaction equation:

$$
\mathrm{O}_{\mathrm{O}}^{\times} \rightarrow \frac{1}{2} \mathrm{O}_{2}+V_{\mathrm{O}} \cdot 2 \mathrm{e}^{\prime}
$$

Thus, oxygen sorption leads to the $\mathrm{Mn}^{2+}$ at tetrahedral sites are easily oxidized to $\mathrm{Mn}^{3+}$. As a result, the configurations of the cation distribution become less stable and easier for migration, which results in the increasing of the resistivity drift. The phenomenon of resistivity drift during the aging test is very consistent with other studies. ${ }^{30,31}$

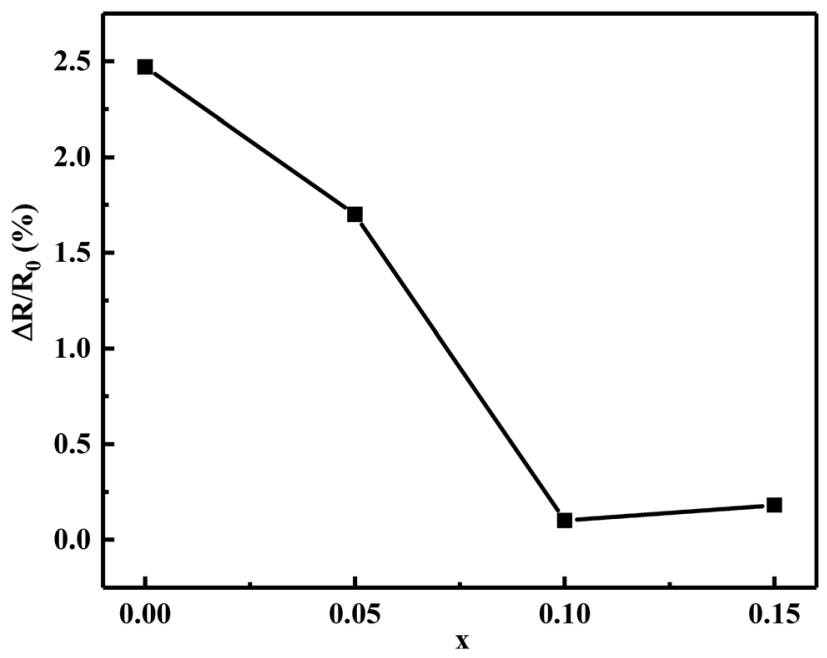

Fig. 8 The relative resistance drift $\Delta R / R_{0}$ of $\mathrm{Bi}_{2} \mathrm{O}_{3}$ content $(0-$ $0.15 \mathrm{wt} \%)$ for the MCF ceramics after the aging test at $125^{\circ} \mathrm{C}$ for $300 \mathrm{~h}$. 
To further clarify the contribution of the grain and grain boundary to the conduction characteristic of MCF ceramics, the AC impedance spectroscopy was engaged in this paper. ${ }^{32-34}$ Fig. 9 shows the simplified equivalent circuit and Nyquist plots for MCF ceramics measured at room temperature with the frequencies range from $100 \mathrm{~Hz}$ to $4 \mathrm{MHz}$. Generally, since the mean relaxation time of grain and grain boundaries differ from each other, two semicircular arcs with different radii can usually be observed in polycrystalline ceramics. In the impedance plane plot, the intercept of the $Z^{\prime \prime}$ axis in high-frequency represents the grain resistance $\left(R_{\mathrm{b}}\right)$, and the low-frequency one depicts the grain boundaries resistance $\left(R_{\mathrm{gb}}\right){ }^{32,35}$ In electrically inhomogeneous ceramics with high grain boundary resistance, however, the impedance plane may not exhibit contributions from grains of the sample. In this paper, for instance, the grain response at high-frequency is hard to observe thoroughly. Only one depressed semicircle in low-frequency observed, which attributed to the contribution of the grain boundaries. It suggests the total impedance of MCF ceramics predominated by grain boundary resistance. It is consistent with other studies. ${ }^{33}$ Moreover, as $\mathrm{Bi}_{2} \mathrm{O}_{3}$ increased from 0 to $0.15 \mathrm{wt} \%$, the variation of grain boundaries resistance $\left(R_{\mathrm{gb}}\right)$ decreased first and then increased, which is similar to the variation of resistivity and can attribute to the oxygen vacancies formed by oxygen adsorption (eqn (5)). It is consistent with elemental mapping (Fig. 5).

Fig. 10 shows the relation of the real part of the impedance $\left(Z^{\prime}\right)$ with frequency. It observed that $Z^{\prime}$ decreases gradually with the increases in frequency means the increased ac conductivity. Further, as $\mathrm{Bi}_{2} \mathrm{O}_{3}$ increased from 0 to $0.15 \mathrm{wt} \%, Z^{\prime}$ decreases first and then increases, which corresponds to the variation of the microstructure and densification. Similar results reported by Zhang et $a l .{ }^{36}$ Fig. 11 presents the relation of the imaginary part
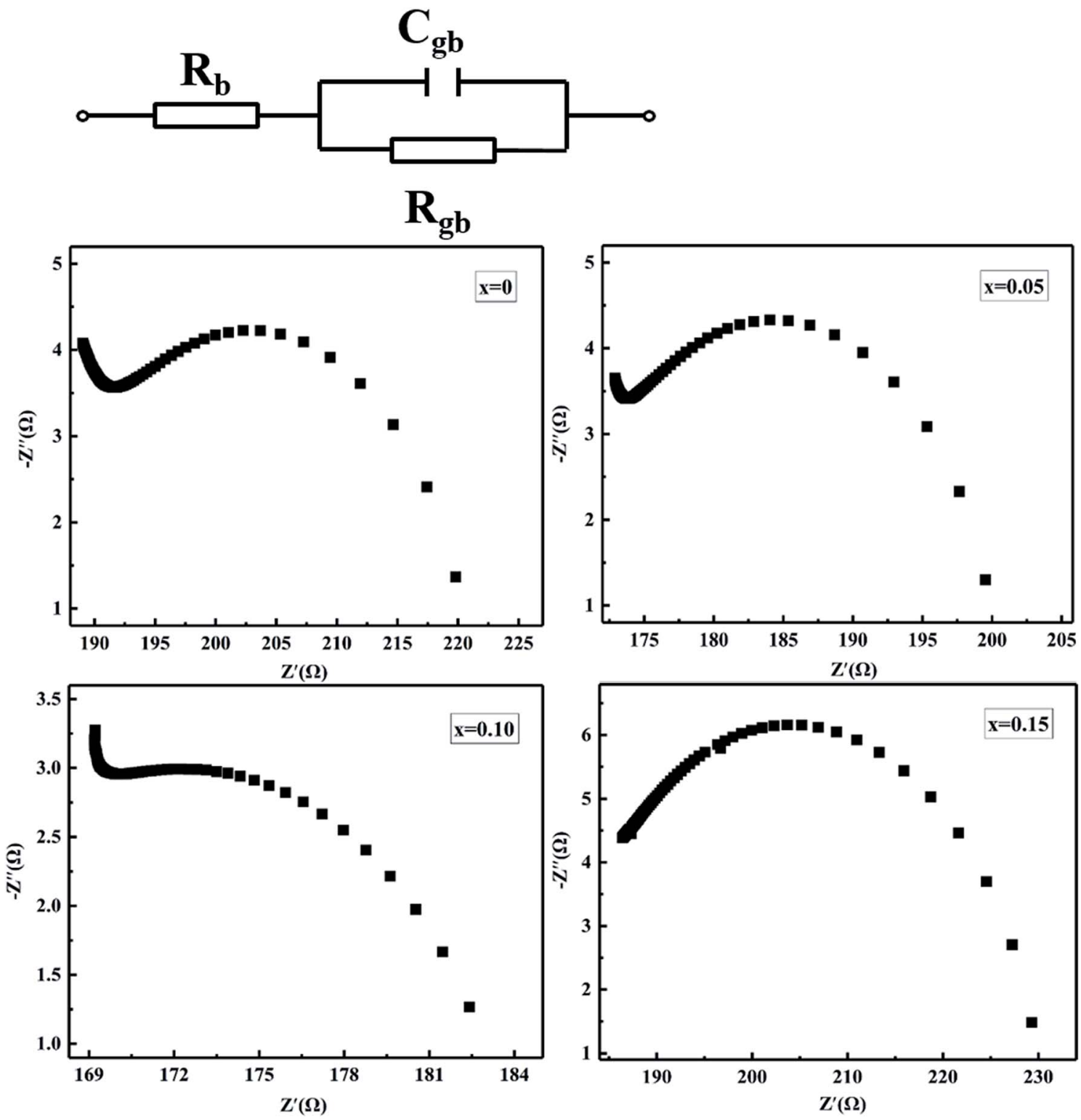

Fig. 9 Simplified equivalent circuit and impedance plots of MCF ceramics with $x$ wt $\% \mathrm{Bi}_{2} \mathrm{O}_{3}$ contents $(0 \leq x \leq 0.15)$. 


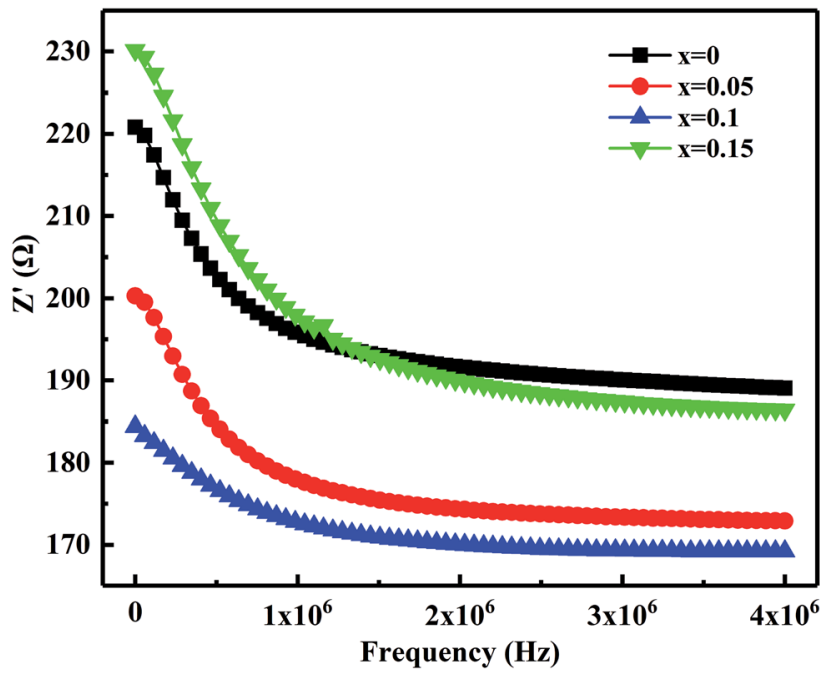

Fig. 10 Variation of the real part of the impedance $\left(Z^{\prime}\right)$ with frequency for MCF ceramics with $x$ wt $\% \mathrm{Bi}_{2} \mathrm{O}_{3}$ contents $(0 \leq x \leq 0.15)$.

$\left(Z^{\prime \prime}\right)$ with frequency. It has observed that $Z^{\prime \prime}$ increases firstly and then decreases, a maximum value appears when the relaxation frequency reached. It indicates that the single relaxation process, which corresponds to the grain boundaries relaxation process. ${ }^{37,38}$ Relaxation behavior induced by the space charge effect in the sample. When it's below the relaxation frequency, the imaginary part, $Z^{\prime \prime}$, is mainly dominated by a long-range motion of charge carriers. Further, above the relaxation frequency, charge carriers are trapped in the potential wells. The imaginary component of the impedance essentially predominated by short-range hopping of charge carriers in potential wells. It's consistent with the impedance data reported in other studies. ${ }^{34}$ Thus, it further confirmed that the resistivity is mainly affected by the relaxation process of grain boundaries.

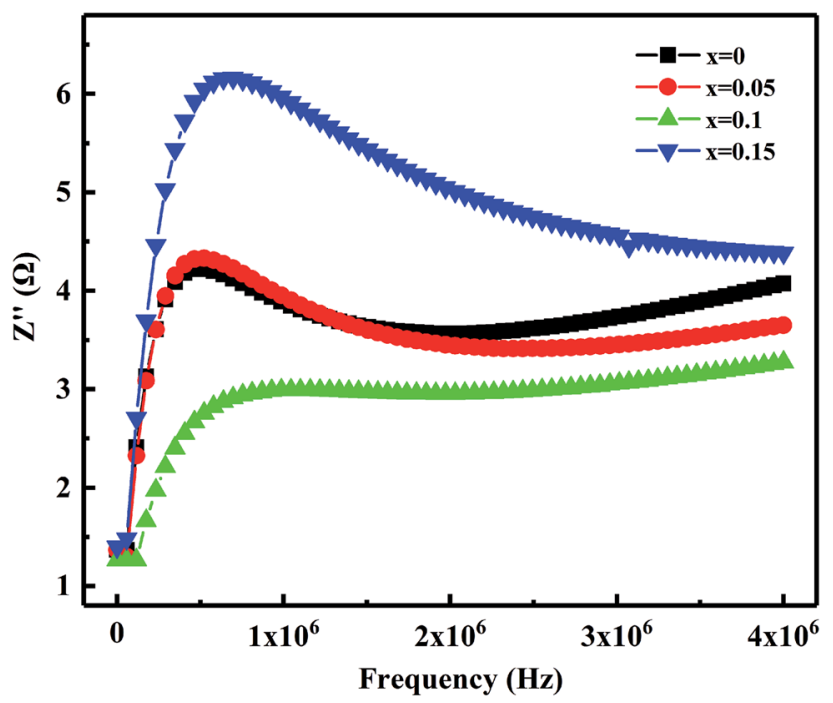

Fig. 11 Variation of the imaginary part of the impedance $\left(Z^{\prime \prime}\right)$ with frequency of MCF ceramics with $x w t \% \mathrm{Bi}_{2} \mathrm{O}_{3}$ contents $(0 \leq x \leq 0.15)$.

\section{Conclusions}

In this paper, the effects of $\mathrm{Bi}_{2} \mathrm{O}_{3}$ additive on the sintering temperature, microstructure, and NTCR characteristics of $\mathrm{Mn}_{1.1} \mathrm{Co}_{1.5} \mathrm{Fe}_{0.4} \mathrm{O}_{4}$ ceramics were systematically investigated. Liquid bismuth trioxide was demonstrated to be an effective agent during sintering of the MCF ceramics. The sintering temperature of the MCF ceramics decreased from $1200{ }^{\circ} \mathrm{C}$ to $1050{ }^{\circ} \mathrm{C}$ by a large amount. Densification and microstructure evolution is mainly due to two different mechanisms in the final stage of sintering. The resistivity decreases first and then increases with increasing $\mathrm{Bi}_{2} \mathrm{O}_{3}$ content. Furthermore, the aging test showed that the $0.1 \mathrm{wt} \% \mathrm{Bi}_{2} \mathrm{O}_{3}$ sample exhibited the lowest resistance drift $\left(\Delta R / R_{0}=0.10 \%\right)$, which can be explained by oxygen sorption. The grain boundary conduction and relaxation behavior have attributed to the oxygen absorption and space charge, respectively. Consequently, it demonstrated that the $\mathrm{Bi}_{2} \mathrm{O}_{3}$ additive is favorable for preparing high-performance NTC ceramics at lower sintering temperatures.

\section{Conflicts of interest}

There are no conflicts to declare.

\section{Acknowledgements}

This work was supported by the National Key Research and Development Program of China (Grant No. 2017YFB0406405).

\section{References}

1 K. C. Feng, P. Y. Chen, C. S. Tu, C. S. Chen, R. R. Chien, C. C. Chiang and W. S. Chang, J. Alloys Compd., 2019, 782, 1094-1102.

2 C. Kai, C. Li, H. Xiang, Y. Tang, Y. Sun and L. Fang, J. Am. Ceram. Soc., 2019, 102, 362-371.

3 Y. Liang, M. Ma, F. Zhang, F. Liu, Z. Liu, D. Wang and Y. Li, Sensors, 2019, 19, 1189.

4 W. Yang, X. Tang, H. Zhang and H. Su, Ceram. Int., 2016, 42, 14609-14613.

5 G. Wang, H. Zhang, X. Huang, F. Xu, G. Gan, Y. Yang, D. Wen, J. Li, C. Liu and L. Jin, Ceram. Int., 2018, 44, 19295-19300.

6 J. Li, D. Wen, Q. Li, T. Qiu, G. Gan and H. Zhang, Ceram. Int., 2018, 44, 678-682.

7 M. Suzuki, J. Phys. Chem. Solids, 1980, 41, 1253-1260.

8 C. Zhao, B. Wang, P. Yang, L. Winnubst and C. Chen, J. Eur. Ceram. Soc., 2008, 28, 35-40.

9 A. Feteira, J. Am. Ceram. Soc., 2009, 92, 967-983.

10 H. Zhang, A. Chang and C. Peng, Microelectron. Eng., 2011, 88, 2934-2940.

11 X. Liu, J. H. Wang, Z. H. Hu, J. C. Yao and A. M. Chang, J. Mater. Sci.: Mater. Electron., 2017, 28, 7243-7247.

12 M. N. Muralidharan, P. R. Rohini, E. K. Sunny, K. R. Dayas and A. Seema, Ceram. Int., 2012, 38, 6481-6486.

13 M. Kong, S. Jiang, T. Xie and H. Zhang, Microelectron. Eng., 2009, 86, 2320-2323. 
14 R. Lebourgeois, S. Duguey, J. P. Ganne and J. M. Heintz, J. Magn. Magn. Mater., 2007, 312, 328-330.

15 W. Ling, H. Zhang, Y. He, Y. Wu, K. Yang, Y. Li and S. Li, J. Magn. Magn. Mater., 2010, 322, 819-823.

16 X. Kuang, G. Carotenuto and L. Nicolais, Adv. Perform. Mater., 1997, 4, 257-274.

17 T. Reimann, J. Topfer, S. Barth, H. Bartsch and J. Muller, Int. J. Appl. Ceram. Technol., 2013, 10, 428-434.

18 K. W. Tay, Y. P. Fu, J. F. Huang and H. C. Huang, Ceram. Int., 2011, 37, 1025-1031.

19 Y. Song, Q. Sun, Y. Lu, X. Liu and F. Wang, J. Alloys Compd., 2012, 536, 150-154.

20 M. R. Tohidifar, Ceram. Int., 2018, 44, 3699-3706.

21 F. Ye, Z. Hou, H. Zhang and L. Liu, J. Am. Ceram. Soc., 2010, 93, 2956-2959.

22 X. He, R. Chu, Z. Xu, Z. Yao and J. Hao, RSC Adv., 2018, 8, 15613-15620.

23 F. Xu, D. Zhang, G. Wang, H. Zhang, Y. Yang, Y. Liao, L. Jin, Y. Rao, J. Li, F. Xie and G. Gan, J. Alloys Compd., 2019, 776, 954-959.

24 R. M. German, P. Suri and S. J. Park, J. Mater. Sci., 2008, 44, 1-39.

25 F. Xie, L. Jia, F. Xu, J. Li, G. Gan and H. Zhang, Ceram. Int., 2018, 44, 13122-13128.

26 F. Xu, Y. Liao, D. Zhang, T. Zhou, J. Li, G. Gan and H. Zhang, Inorg. Chem., 2017, 56, 4512-4520.
27 A. R. Uhl, P. Fuchs, A. Rieger, F. Pianezzi, et al., Prog. Photovolt. Res. Appl., 2015, 23, 1110-1119.

28 G. Wang, H. Zhang, C. Liu, H. Su, L. Jia, J. Li, X. Huang and G. Gan, J. Electron. Mater., 2018, 47, 4672-4677.

29 X. Wang, Y. Li, Z. Chen, H. Zhang, H. Su, G. Wang, Y. Liao and Z. Zhong, J. Alloys Compd., 2019, 797, 566-572.

30 D. F. Li, S. X. Zhao, K. Xiong, H. Q. Bao and C. W. Nan, J. Alloys Compd., 2014, 582, 283-288.

31 C. J. Ma, Y. F. Liu, Y. N. Lu, H. Gao, H. Qian and J. X. Ding, Mater. Sci. Eng., B, 2014, 188, 66-71.

32 S. G. Song, Z. Ling and F. Placido, Mater. Res. Bull., 2005, 40, 1081-1093.

33 Z. Song, S. Zhang, H. Liu, H. Hao, M. Cao, Q. Li, Q. Wang, Z. Yao, Z. Wang, M. T. Lanagan and J. Jones, J. Am. Ceram. Soc., 2015, 98, 3212-3222.

34 Z. Peng, X. Zeng, F. Cao and X. Yang, J. Alloys Compd., 2017, 695, 626-631.

35 J. X. Wang, H. Zhang, X. Sun, Y. Liu and Z. C. Li, J. Mater. Sci.: Mater. Electron., 2016, 27, 11902-11908.

36 H. Zhang, T. Liu, L. Zhao, H. Jiang and A. Chang, J. Mater. Sci.: Mater. Electron., 2017, 28, 14195-14201.

37 K. Lily, K. Kumari, K. Prasad and K. L. Yadav, J. Mater. Sci., 2007, 42, 6252-6259.

38 B. Zhang, Q. Zhao, A. Chang, J. Yao, P. Zhao, F. Guan and W. Kong, J. Alloys Compd., 2012, 512, 132-139. 